> La fièvre typhoïde est une maladie sévère et l'introduction d'une antibiothérapie efficace par le chloramphénicol en 1948 a été un progrès considérable. Dès les années suivantes, des souches de Salmonella enterica sérotype Typhi résistantes à cet antibiotique ont été isolées. Ce même phénomène s'est produit après l'introduction des aminopénicillines et du cotrimoxazole. Au cours de la deuxième moitié des années 1980, des souches multirésistantes (MR) à ces trois classes d'antibiotiques ont émergé puis envahi le sous-continent indien et l'Asie du Sud-Est. Dans les années 1990, toujours en Asie, ces souches MR sont devenues résistantes à l'acide nalidixique avec une sensibilité diminuée à la ciprofloxacine $\left(\mathrm{Cip}^{\mathrm{SD}}\right)$, vraisemblablement secondaire à I'utilisation massive de fluoroquinolones. Ces souches $\mathrm{Cip}^{\mathrm{SD}}$ qui peuvent être la cause de mauvaises réponses thérapeutiques, voire d'échecs, avec les fluoroquinolones, sont d'identification difficile au laboratoire. Seule une détermination de la CMI (concentration minimale inhibitrice) de la ciprofloxacine permet un diagnostic de certitude (CMI comprise entre 0,125 et $1 \mathrm{mg} / \mathrm{I}$ ). Récemment, quelques souches hautement résistantes aux traitements de première intention, fluoroquinolones et céphalosporines de troisième génération, ont été identifiées, toujours en provenance d'Asie. <

\section{La fièvre typhoïde n'est plus aussi simple à soigner}

\author{
François-Xavier Weill
}

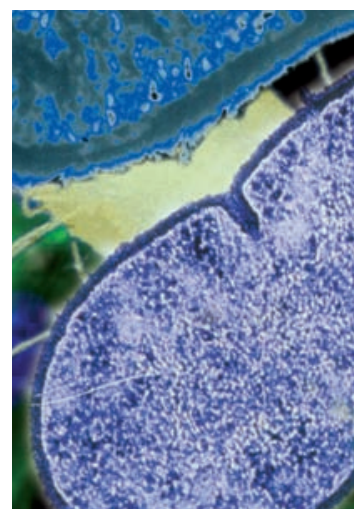

Institut Pasteur, Centre national de référence des Salmonella, unité des bactéries pathogènes entériques, 28 , rue du docteur Roux, 75724 Paris cedex 15, France. fxweill@pasteur.fr

bien indirecte par l'intermédiaire d'eau ou d'aliments également souillés par des matières fécales contaminées. Une particularité de Salmonella Typhi est qu'elle peut, au décours d'une infection, persister de façon asymptomatique dans le tube digestif (réensemencement à partir de la vésicule biliaire) et, beaucoup plus rarement, dans les voies urinaires de certains individus appelés porteurs sains. Ceux-ci ont un rôle important dans la transmission de la maladie comme en témoignent les exemples célèbres du laitier $N$. the milker, qui infecta entre 1893 et 1909 plus de deux cents personnes en Angleterre, ou de la cuisinière Mary Mallon, surnommée Marie Typhoïde, au début du XXe siècle aux États-Unis [1]. Du fait des caractéristiques épidémiologiques de cette infection, la fièvre typhoïde a été une cause importante de morbidité et de mortalité en Europe, et ce jusqu'à la première moitié du XXe siècle. L'accès à une eau potable, l'assainissement (fosse septique, tout-à-l'égout), les règles d'hygiène de base (lavage des mains) et de sécurité alimentaire, associés à un traitement antibiotique efficace des patients infectés ont permis de faire quasiment disparaître les cas autochtones de fièvre typhoïde en France dans la période d'après-guerre [2]. Quelques cas sporadiques ou de petites microépidémies sont néanmoins toujours détectés en relation avec des porteurs sains originaires de pays d'endémie et travaillant dans le secteur de la restauration. Si la quasi-disparition de la fièvre typhoïde a été observée en Europe de l'Ouest et en Amérique du Nord, cette maladie reste très prévalente dans le reste du monde et en particulier en Asie et en 
Afrique. La morbidité et la mortalité de la fièvre typhoïde avaient été estimées à l'échelle planétaire respectivement à 21 millions de cas annuels et 200000 décès au début des années 2000 [3].

\section{Historique de la résistance aux antibiotiques}

La fièvre typhoïde est une maladie sévère et l'introduction d'une antibiothérapie efficace par le chloramphénicol à la fin des années 1940 a été un progrès considérable puisque le taux de mortalité est passé de $10 \%$ et plus à $1 \%$ voire moins [4]. Cependant, en plus des risques hématologiques liés au traitement ${ }^{1}$, des souches résistantes au chloramphénicol, isolées de cas sporadiques, ont été publiées dès 1950 en Angleterre puis dans les années 1960 en Inde, au Chili, en Afrique de l'Ouest, en Israël, au Yémen et en Grèce [5]. La résistance au chloramphénicol de certaines souches était transférable d'une bactérie à l'autre grâce à un élément extra-chromosomique appelé facteur $\mathrm{R}$ (pour resistance factor), connu aujourd'hui sous le nom de plasmide. À la fin des années 1960, des souches multirésistantes à l'ampicilline, au chloramphénicol et aux tétracyclines ont été identifiées en Égypte, au Yémen et au Pakistan, mais toujours à partir de cas sporadiques [5]. La résistance à ces trois antibiotiques était portée par un plasmide de type hcF (typage par la méthode d'incompatibilité), probablement acquis à partir de bactéries commensales du tube digestif, comme Escherichia coli.

Les premières épidémies de fièvre typhoïde causées par des souches multirésistantes (au chloramphénicol, à la streptomycine, aux sulfamides et aux tétracyclines ou type « CSSuTe ») eurent lieu au Mexique et en Inde au cours de l'année 1972 [4, 6, 7]. L'épidémie mexicaine était de grande importance avec plus de 10000 cas documentés. Dans les deux cas, la résistance était liée à la présence d'un plasmide de type IncHIl. À partir de 1972, des souches épidémiques similaires sont alors mises en évidence au Vietnam et en Thaïlande $[4,8,9]$. Il est à noter que vers la fin de l'épidémie mexicaine, quelques souches portaient une résistance additionnelle à l'ampicilline apportée par différents types de plasmides appartenant aux groupes IncA, IncC ou Incl. Cependant, cette résistance n'a été que provisoire du fait peut être de l'instabilité de ces plasmides. Le triméthoprime-sulfaméthoxazole (cotrimoxazole) a alors été utilisé avec succès malgré l'identification de cas très sporadiques de souches résistantes à cet antibiotique [10]. En 1981 au Pérou, au décours d'une épidémie causée par des souches de type CSSuTe qui avait débuté en 1979, 47,2\% des souches étaient devenues également résistantes au cotrimoxazole et 21 \% à l'ampicilline [10]. Des plasmides de type IncHIl de $180 \mathrm{~kb}$ transféraient en bloc les déterminants de résistance. Cependant, aucune souche épidémique portant la résistance simultanément aux trois antibiotiques de première intention (chloramphénicol, aminopénicillines et cotrimoxazole) n'avait été encore mise en évidence. De telles souches multirésistantes (MR) finissent par émerger dans le sous-continent indien et en Asie du Sud-Est au cours de la deuxième moitié des années 1980 [11]. Le support de cette triple résistance était toujours un plasmide de grande

${ }^{1}$ Il s'agit le plus souvent d'agranulocytose. taille appartenant au groupe d'incompatibilité HIl. La dissémination de ces souches au cours des années 1990 $a$ alors été très rapide [11]. Au Vietnam, par exemple, la première souche MR a été détectée dans le sud du pays en 1992-1993 et entre 1995 et 2002 plus de $80 \%$ des souches étaient devenues MR [12]. L'utilisation des fluoroquinolones dans le traitement de la fièvre typhoïde a donc débuté à partir de la fin des années 1980 dans le sud-est asiatique en réponse à l'émergence des souches MR [11]. La première souche MR avec une résistance additionnelle à l'acide nalidixique $\left(M R-N a l^{R}\right)$ décrite dans la littérature a été isolée en 1991 en Grande-Bretagne chez un enfant rentrant $d^{\prime}$ Inde [13]. Ces souches $M R-N a l^{R}$ avaient également une sensibilité diminuée à la ciprofloxacine ( $\mathrm{Cip}^{\mathrm{SD}}$ ) avec des CMI comprises entre 0,125 et 0,5 mg/l (alors que les souches sauvages ont des CMI comprises entre 0,002 et $0,06 \mathrm{mg} / \mathrm{l}$ ) et elles se sont par la suite propagées dans le sous-continent indien et dans le sud-est asiatique. Elles ont été responsables d'épidémies (notamment au Tadjikistan et au Vietnam) et de mauvaises réponses voire d'échecs thérapeutiques après traitement par des fluoroquinolones [11, 14-18]. Ces constatations cliniques ont été par la suite corroborées par des données pharmacocinétiques et pharmacodynamiques [19]. La probabilité d'atteindre une valeur prédictive d'efficacité (rapport aire sous la courbe des concentrations sériques/CMI > 105) était de plus de $90 \%$ pour une souche ayant une CMI de 0,12 mg/I pour la ciprofloxacine mais chutait à $5,4 \%$ pour une souche ayant une $C M I$ de $0,25 \mathrm{mg} / \mathrm{l}$. La dissémination des souches MR-Nal ${ }^{\mathrm{R}}-\mathrm{Cip}^{\mathrm{SD}}$ a été très rapide par exemple au Vietnam, où elles sont apparues à partir de 1993, représentant alors $4 \%$ des souches de Salmonella Typhi pour atteindre $12 \%$ en 1997 , et $80 \%$ au-delà [14]. Une étude de génétique des populations bactériennes a mis en évidence que ces souches $\mathrm{Nal}^{\mathrm{R}}$ appartenaient à un même clone d'origine asiatique nommé $\mathrm{H} 58$ et porteur d'une mutation dans le gène gyrA codant pour la sous-unité A de l'ADN gyrase?, cible des quinolones [1]. De façon intéressante et sans qu'aucun lien n'ait été jamais évoqué, entre 1993 et 1995, plusieurs essais de traitements courts ( 5 jours à $400 \mathrm{mg} / \mathrm{j}$ puis 3 jours à $10 \mathrm{mg} / \mathrm{kg} / \mathrm{j}$ voire 2 jours à $15 \mathrm{mg} /$ $\mathrm{kg} / \mathrm{j}$ chez les enfants) à base d'ofloxacine par voie orale chez des patients atteints de fièvre typhoïde non compliquée ont été conduits au Vietnam [20-22]. Les fluoroquinolones par voie orale s'étaient révélées plus efficaces que la ceftriaxone par voie intraveineuse lors

2 L'ADN gyrase participe chez les bactéries aux mécanismes de réplication, transcription et recombinaison. L'ouverture de l'ADN va provoquer des supertours qui vont être éliminés par l'ADN gyrase (topo-isomérase). 


\begin{tabular}{|c|c|c|c|c|c|c|c|c|}
\hline \multirow[t]{2}{*}{ Antibiotique } & \multicolumn{8}{|c|}{ Pourcentage de souches résistantes } \\
\hline & $\begin{array}{c}1997 \\
(n=40) \\
(N=170)\end{array}$ & $\begin{array}{c}2000 \\
(n=40) \\
(N=186)\end{array}$ & $\begin{array}{c}2002 \\
(n=40) \\
(N=133)\end{array}$ & $\begin{array}{c}2005 \\
(n=63) \\
(N=116)\end{array}$ & $\begin{array}{c}2006 \\
(n=106) \\
(N=111)\end{array}$ & $\begin{array}{c}2007 \\
(n=65) \\
(N=65)\end{array}$ & $\begin{array}{c}2008 \\
(n=90) \\
(N=90)\end{array}$ & $\begin{array}{c}2009 \\
(n=120) \\
(N=120)\end{array}$ \\
\hline Amoxicilline & 0 & 0 & 2,5 & 8,1 & 12,3 & 20 & 12,2 & 16,7 \\
\hline Ceftriaxone/ceftazidime & 0 & 0 & 0 & 0 & 0 & 0 & 0 & 0 \\
\hline Acide nalidixique & 0 & 5 & 7,5 & 17,8 & 37,7 & 33,3 & 27,8 & 23,3 \\
\hline Ciprofloxacine & 0 & 0 & 0 & 0 & 0 & 0 & 0 & 0 \\
\hline Cotrimoxazole & 5 & 2,5 & 7,5 & 7,9 & 12,3 & 22,2 & 12,2 & 19,2 \\
\hline Chloramphénicol & 7,5 & 0 & 7,5 & 5,9 & 12,3 & 15,6 & 14,4 & 18,3 \\
\hline Tétracycline & 5 & 0 & 7,5 & 5,9 & 12,3 & 15,6 & 8,9 & 10,8 \\
\hline
\end{tabular}

Tableau I. Résistance aux antibiotiques de S. enterica sérotype Typhi, France, 1997-2009. Souches isolées en France métropolitaine (une seule par patient). $n$ : nombre de souches étudiées; $N$ : nombre de souches reçues au CNR-Salm. Données extraites du rapport annuel du CNR des Salmonella téléchargeable à l'adresse suivante : http://www.pasteur.fr/ip/portal/action/WebdriveActionEvent/oid/01s-00003k-03o.

d'infections par des souches sensibles aux antibiotiques [20]. L'accès facile aux fluoroquinolones, et ce en dehors de toute prescription médicale, a été certainement un facteur favorisant la dissémination de ce clone H58 en Asie [14, 23].

\section{La résistance aux antibiotiques de nos jours}

\section{Distribution des souches résistantes}

Le plus souvent, les pays où la fièvre typhoïde sévit de façon endémique ne disposent pas d'un système national de surveillance microbiologique de cette infection et donc ne disposent pas de données stables en ce qui concerne la sensibilité aux antibiotiques des souches circulantes permettant d'édicter des recommandations pour leur traitement empirique. Pour avoir une idée de la circulation de ces souches à l'échelle de la planète, il faut s'intéresser à des études locales, le plus souvent limitées dans le temps et dont la représentativité n'est pas toujours optimale, ou bien alors se reposer sur des réseaux de surveillance européens ou américains qui rapportent les données issues de voyageurs ou d'immigrants [24, 25] (Tableau I). Une étude portant sur plus de 2000 souches isolées aux États-Unis entre 1999 et 2006 a montré que la proportion de souches MR avait fluctué entre $6 \%$ (2002) et $19 \%$ (2001 et 2006) alors que celle des Nal était en constante augmentation, de $19 \%$ en 1999 à $54 \%$ en 2006 [25]. Parmi les destinations à risque pour l'acquisition de souches résistantes figurait en premier lieu le sous-continent indien : $17 \%$ des patients y ayant séjourné ont été infectés par une souche MR et $65 \%$ par une souche $\mathrm{Nal}^{\mathrm{R}}$-Cip ${ }^{\mathrm{SD}}$ [25]. En 2006, $88 \%$ des isolats indiens étaient $\mathrm{Nal}^{\mathrm{R}}{ }_{-}$ $\mathrm{Cip}^{\mathrm{SD}}$ [25]. Pour les patients ayant séjourné en Asie du Sud-Est, ces pourcentages étaient respectivement de $14 \%$ et $20 \%$ pour les souches $M R$ et $\mathrm{Nal}^{\mathrm{R}}$-Cip ${ }^{\mathrm{SD}}$, et pour ceux ayant séjourné en Afrique, ils étaient de $24 \%$ et $0 \%$. Les plus faibles pourcentages d'infection par des souches résistantes étaient obtenus en Amérique centrale et du Sud avec $1 \%$ de patients infectés par des souches MR et $3 \%$ par des souches $\mathrm{Nal}^{\mathrm{R}}-\mathrm{Cip}^{\mathrm{SD}}$. Les données anglaises sur 692 souches isolées entre 2000 et 2003 confirment la forte association entre souches résistantes et souscontinent indien [24]. Seuls $23 \%$ des souches acquises en Inde étaient sensibles à l'ensemble des antibiotiques.

Les données françaises provenant du Centre national de référence des Salmonella sont indiquées dans le Tableau I. Ces données globales sont toutefois à pondérer en fonction du lieu d'acquisition de la bactérie. Ainsi en 2008-2009, toutes les souches (dont l'origine géographique était connue) acquises en Afrique du Nord $(n=29)$ et en Amérique centrale ou du Sud $(n=7)$ étaient sensibles aux antibiotiques alors que celles acquises en Asie $(n=50)$ étaient MR $(2 \%, 1$ sur 50$)$, $\mathrm{MR}-\mathrm{Nal}{ }^{\mathrm{R}}-\mathrm{Cip}^{\mathrm{SD}}(30 \%, 15$ sur 50$)$ ou uniquement $\mathrm{Nal}^{\mathrm{R}_{-}}$ Cip $^{\text {SD }}(54 \%, 27$ sur 50$)$. Seuls $15 \%$ ( 7 sur 50 ) des souches étaient sensibles aux antibiotiques. II est à noter que 48 souches sur les 50 provenaient du sous-continent indien. Les données locales asiatiques ou les données issues de voyageurs ou d'immigrants confirment que les souches $\mathrm{Nal}^{\mathrm{R}}-\mathrm{Cip}^{\mathrm{SD}}$ sont maintenant prédominantes dans le sous-continent indien et au Vietnam mais qu'elles recouvrent leur sensibilité aux anciens antibiotiques de première ligne que sont le chloramphénicol, les aminopénicillines et le cotrimoxazole [14, 24-26]. L'explication la plus vraisemblable en est l'élimination progressive du plasmide de multirésistance probablement due à la baisse de la pression de sélection par ces 
antibiotiques qui sont moins utilisés. Concernant l'Afrique de l'Ouest et l'Afrique centrale, des souches MR y sont de plus en plus fréquemment identifiées chez des voyageurs ou des immigrants, leur proportion atteignant $30 \%$ (11 sur 37 ) en 2008-2009. De telles souches MR ont été également décrites localement dans certaines zones géographiques comme le Kenya, le Ghana, le Nigéria, le Cameroun et l'Égypte ([11] et Weill FX, données personnelles). Les souches MR de l'Afrique de l'Ouest et la majorité de celles d'Afrique centrale n'appartiennent pas au clone asiatique $\mathrm{H} 58$ mais auraient été sélectionnées à partir de souches locales (Weill FX, données personnelles). Les souches $\mathrm{Nal}^{\mathrm{R}}$ $\mathrm{Cip}^{\mathrm{SD}}$ sont en revanche exceptionnelles en Afrique [1].

\section{Problèmes posés par l'identification des souches résistantes}

Ces souches $\mathrm{Nal}^{\mathrm{R}}$-Cip ${ }^{\mathrm{SD}}$ représentent donc actuellement la principale population résistante à laquelle nous sommes confrontés et elle pose de sérieux problèmes de prise en charge thérapeutique. Cependant, ces souches ne sont certainement pas identifiées de façon optimale par les laboratoires de microbiologie clinique du fait de l'inadéquation des concentrations critiques des fluoroquinolones édictées par les sociétés savantes telles que le comité de l'antibiogramme de la Société française de microbiologie (CA-SFM) ou le Clinical and laboratory standards institute (CLSI). Le CA-SFM recommande pour les entérobactéries les concentrations critiques suivantes pour la ciprofloxacine: $C M I \leq 0,5 \mathrm{mg} / \mathrm{I}$ pour définir la sensibilité et $\geq 1 \mathrm{mg} / /$ pour la résistance ${ }^{3}$ alors que le CLSI recommande $\leq 1 \mathrm{mg} /$ / pour la sensibilité et $\geq 4 \mathrm{mg} / \mathrm{l}$ pour la résistance, et ce malgré plusieurs articles publiés dans la littérature demandant une révision de ces concentrations critiques [16, 17]. En utilisant de telles concentrations critiques, toutes les souches Cip $^{\text {SD }}$ sont alors définies comme sensibles à la ciprofloxacine. Cependant, le CLSI préconise depuis 2004 d'utiliser le test de sensibilité à l'acide nalidixique sur les souches de salmonelles extra-intestinales et, en cas de résistance à cet antibiotique et malgré la sensibilité à la ciprofloxacine, de prévenir le clinicien du risque de mauvaise réponse au traitement par les fluoroquinolones, voire d'échec thérapeutique. Le CA-SFM préconise quant à lui depuis 2010, devant une résistance à l'acide nalidixique, de considérer toute souche de Salmonella comme résistante aux fluoroquinolones (CA-SFM). Ce test de dépistage des souches Cip ${ }^{S D}$ fondé sur la réponse à l'acide nalidixique a été proposé dès le milieu des années 1990 [15]. II repose sur le fait que la très grande majorité des souches Cip $^{\text {SD }}$ circulantes avaient une mutation dans le codon 83 ou 87 du gène gyr $A$, ce qui a pour conséquence de conférer une résistance importante à l'acide nalidixique (CMI $\geq 128 \mathrm{mg} / \mathrm{I})$. Cependant, plusieurs publications récentes ont montré l'existence d'une population Cip ${ }^{\text {SD }}$ qui conservait une sensibilité à l'acide nalidixique avec des CMI entre 4 et $16 \mathrm{mg} /$ ( (la résistance pour cet antibiotique est définie par une CMI supérieure à $16 \mathrm{mg} / \mathrm{I}$ pour le (A-SFM) $[18,24,25,27]$. Cette population originaire d'Asie, et plus particulièrement du sous-continent indien, n'est donc pas détectable par le test à l'acide nalidixique. Cela remet donc en cause ce test car les souches $\mathrm{Nal}^{\mathrm{S}}$-Cip ${ }^{\mathrm{SD}}$ représentaient $11,6 \%$ (49 sur 421) des souches de Salmonella Typhi isolées au Royaume-Uni

\footnotetext{
${ }^{3}$ http://www.sfm.asso.fr/nouv/general.php?pa=2.
}

entre 1999 et 2003 et $5 \%$ (36 sur 770) des souches isolées aux États-Unis entre 1999 et 2006 [24, 25]. En France, une étude rétrospective sur les souches du CNR a retrouvé une prévalence plus faible à $1 \%$ (7 sur 685), ce qui peut être expliqué par un moindre flux de population entre notre pays et le sous-continent indien en comparaison avec ceux induits par les relations historiques entre le Royaume-Uni et ce dernier. Le mécanisme de résistance de ces souches a été récemment mis en évidence, il s'agit de mutations dans le codon 464 du gène gyr $B$ codant pour la sous-unité B de l'ADN gyrase $[27,28]$. Pour conclure sur les moyens méthodologiques d'identification de ces souches Cip ${ }^{S D}$, seule la détermination de la CMI des fluoroquinolones et en particulier celle de la ciprofloxacine, est à même de les identifier formellement. II conviendrait que toutes les souches ayant une CMI comprise entre 0,125 et $1 \mathrm{mg} / \mathrm{l}$ soient considérées désormais comme de sensibilité diminuée et qu'un commentaire mentionnant le risque d'échec thérapeutique en cas d'utilisation de fluoroquinolones aux doses habituelles accompagne le résultat.

\section{Choix de l'antibiothérapie}

L'organisation mondiale de la santé (OMS) recommandait en 2003 l'utilisation en première intention de fluoroquinolones (ofloxacine ou ciprofloxacine) ou de céfixime pour le traitement des fièvres typhoïdes non compliquées causées par des souches MR, et l'azithromycine, les céphalosporines de troisième génération (C3G) telle que la ceftriaxone ou une fluoroquinolone à forte dose pendant 10 à 14 jours pour les infections par des souches Cip ${ }^{\text {SD }}$ (Tableau II). Dans une étude randomisée portant sur 125 patients vietnamiens infectés essentiellement par des souches MR-Nal ${ }^{R}-\mathrm{Cip}^{\mathrm{SD}}$, I'ofloxacine utilisée à la dose de $20 \mathrm{mg} / \mathrm{kg} / \mathrm{j}$ pendant 7 jours ne permettait de guérir que $64 \%$ des patients et la défervescence thermique était obtenue en 8 jours en moyenne [29]. Au cours de la même étude, l'azithromycine utilisée à la dose de $10 \mathrm{mg} / \mathrm{kg} / \mathrm{j}$ pendant 7 jours permettait la guérison de $82 \%$ des patients (défervescence thermique obtenue en 6 jours). Un traitement hybride (ofloxacine à $15 \mathrm{mg} / \mathrm{kg} / \mathrm{j}$ pendant 7 jours et azithromycine à $10 \mathrm{mg} / \mathrm{kg} / \mathrm{j}$ pendant les 3 premiers jours) donnait des résultats intermédiaires. Deux autres essais randomisés réalisés au Vietnam entre 2004 et 2005 (285 patients) et au Népal en 2005 (158 patients) chez des patients atteints de fièvre typhoïde non compliquée et principalement infectés par des souches Cip ${ }^{S D}$ ont concerné la gatifloxacine $(10 \mathrm{mg} / \mathrm{kg} / \mathrm{j}$ pendant 7 jours $)$ versus le céfixime (20 mg/kg/j pendant 7 jours) ou l'azithromycine (20 mg/kg/j pendant 7 jours) [30, 31]. La gatifloxacine est une nouvelle fluoroquinolone qui a démontré une 


\begin{tabular}{|c|c|c|c|c|c|c|}
\hline $\begin{array}{l}\text { Sensibilité } \\
\text { des souches }\end{array}$ & Antibiotique & $\begin{array}{l}\text { Dose } \\
\text { journalière } \\
\text { en } \mathrm{mg} / \mathrm{kg}\end{array}$ & $\begin{array}{l}\text { Durée } \\
\text { en jours }\end{array}$ & Antibiotique & $\begin{array}{l}\text { Dose } \\
\text { journalière } \\
\text { en } \mathbf{m g} / \mathbf{k g}\end{array}$ & $\begin{array}{l}\text { Durée } \\
\text { en jours }\end{array}$ \\
\hline \multicolumn{7}{|c|}{ Fièvre typhoïde non compliquée } \\
\hline Sensible & $\begin{array}{l}\text { Fluoroquinolone (ofloxacine } \\
\text { ou ciprofloxacine) }\end{array}$ & 15 & 5 à 7 & $\begin{array}{l}\text { Chloramphénicol } \\
\text { Amoxicilline } \\
\text { Cotrimoxazole }\end{array}$ & $\begin{array}{c}50-75 \\
75-100 \\
8-40\end{array}$ & $\begin{array}{c}14-21 \\
14 \\
14\end{array}$ \\
\hline MR & $\begin{array}{l}\text { Fluoroquinolone ou } \\
\text { Céfixime }\end{array}$ & $\begin{array}{c}15 \\
15-20\end{array}$ & $\begin{array}{l}5 \text { à } 7 \\
7 \text { à } 14\end{array}$ & $\begin{array}{l}\text { Azithromycine } \\
\text { Céfixime }\end{array}$ & $\begin{array}{l}8-10 \\
15-20\end{array}$ & $\begin{array}{c}7 \\
7-14\end{array}$ \\
\hline $\mathrm{Nal}^{\mathrm{R}}$ & $\begin{array}{l}\text { Azithromycine ou } \\
\text { Ceftriaxone }\end{array}$ & $\begin{array}{l}8-10 \\
75\end{array}$ & $\begin{array}{c}7 \\
10 \text { à } 14\end{array}$ & Céfixime & 20 & $7-14$ \\
\hline Fièvre typhoïde $s$ & & & & & & \\
\hline Sensible & Fluoroquinolone & 15 & 10 à 14 & $\begin{array}{l}\text { Chloramphénicol } \\
\text { Amoxicilline } \\
\text { Cotrimoxazole }\end{array}$ & $\begin{array}{l}50-75 \\
75-100 \\
8-40\end{array}$ & $\begin{array}{c}14-21 \\
14 \\
14\end{array}$ \\
\hline MR & Fluoroquinolone & 15 & 10 à 14 & $\begin{array}{l}\text { Ceftriaxone ou } \\
\text { Céfotaxime }\end{array}$ & $\begin{array}{l}60 \\
80\end{array}$ & $\begin{array}{l}10-14 \\
10-14\end{array}$ \\
\hline $\mathrm{Nal}^{\mathrm{R}}$ & $\begin{array}{l}\text { Ceftriaxone ou } \\
\text { Céfotaxime }\end{array}$ & $\begin{array}{l}60 \\
80\end{array}$ & $\begin{array}{l}10 \text { à } 14 \\
10 \text { à } 14\end{array}$ & Fluoroquinolone & 20 & 14 \\
\hline
\end{tabular}

Tableau II. Recommandations pour le traitement de la fièvre typhoïde d'après l'OMS et [40]. Le rapport de l'OMS est téléchargeable à l'adresse suivante: http://whqlibdoc.who.int/hq/2003/WHO_V\&B_03.07.pdf.

activité bactéricide rapide sur les souches $\mathrm{Cip}^{\mathrm{SD}}$ et dont les CMI sont un peu plus faibles que celles de la ciprofloxacine sur ces mêmes souches $\mathrm{Cip}^{\mathrm{SD}}[26,31]$. Au cours de ces deux essais, l'échec thérapeutique était documenté pour $3,5 \%$ à $9 \%$ des patients traités par la gatifloxacine (défervescence thermique obtenue entre 92 et 106 heures en moyenne), $9 \%$ des patients traités par l'azithromycine (défervescence thermique obtenue en 106 heures), et $37,6 \%$ des patients traités par le céfixime (défervescence thermique obtenue en 138 heures). Ces protocoles thérapeutiques qui répondent à des impératifs de pays d'endémie (disponibilité de la molécule, facilité d'administration, faible coût du traitement) ne sont toutefois pas transposables dans leur intégralité dans nos pays occidentaux. Les essais thérapeutiques plus en phase avec la pratique occidentale (par exemple des combinaisons ciprofloxacineC3G par voie parentérale) sont quant à eux très difficiles à réaliser du fait du faible nombre des cas d'importation.

De façon pratique, il est donc clair que le traitement empirique (en attendant la confirmation de l'analyse de la sensibilité aux antibiotiques par le laboratoire) doit se baser principalement sur l'origine géographique de la contamination. Pour les patients adultes contaminés en dehors de l'Asie, les fluoroquinolones peuvent être utilisées en première intention et poursuivies si la souche isolée est bien sensible à la ciprofloxacine $(\mathrm{CMI} \leq 0,06 \mathrm{mg} / \mathrm{l})$. Pour les contaminations par des souches asiatiques, Gaborieau et al. [27] ont proposé un traitement initial à base de C3G par voie parentérale à fortes doses $(75 \mathrm{mg} / \mathrm{kg} / \mathrm{j}$, sans dépasser $4 \mathrm{~g} / \mathrm{j}$, en une seule injection), avec relais par une fluoroquinolone (ciprofloxacine à $15 \mathrm{mg} / \mathrm{kg} / \mathrm{j}$ en deux doses pendant 7 jours) en cas de sensibilité microbiologique avérée de la souche. Bien que le choix de la ciprofloxacine par rapport à l'ofloxacine ne repose sur aucune étude bioclinique, la ciprofloxacine semble plus utilisée peut être en raison de sa posologie plus forte, de son élimination en partie biliaire, des CMI plus basses que celles de l'ofloxacine et finalement de l'utilisation abondante de critères basés sur la CMI de la ciprofloxacine dans les publications internationales. En cas d'infection par une souche non sensible aux fluoroquinolones, s'il s'agit d'une forme clinique non compliquée, un relais par azithromycine ( $1 \mathrm{~g}$ le premier jour puis 8 à $10 \mathrm{mg} / \mathrm{kg} / \mathrm{j}$ en une dose pendant 7 jours) a été proposé [27]. 
Dans le cas d'une fièvre typhoïde «autochtone», on peut parfois suspecter l'origine géographique probable de la souche si l'infection est survenue au décours d'un repas communautaire ou dans un restaurant exotique ou à la suite de contacts avec des personnes vivant ou ayant vécu en zone d'endémie. En revanche, si la contamination a eu lieu dans un restaurant traditionnel, il est souvent très difficile de s'orienter avant les résultats de l'enquête épidémiologique, car de nombreux travailleurs du monde de la restauration sont originaires de zones d'endémie, que ce soit d'Afrique ou d'Asie.

\section{La résistance aux antibiotiques de demain}

Du fait de la capacité d'adaptation des bactéries à leur environnement, il est évident que la résistance de Salmonella Typhi va progressivement s'étendre à des molécules actuellement utilisées en première intention. Ainsi, des souches hautement résistantes à la ciprofloxacine (CMI $\geq 4 \mathrm{mg} / \mathrm{I})$ originaires du sous-continent indien ont été identifiées ; elles restent toutefois rares puisqu'elles ne représentaient que 0,2\% (5 sur 2016) des souches isolées aux États-Unis entre 1999 et 2006 [25, 26, 32, 33]. Une seule souche de ce type a été identifiée en France depuis 1997. Ces souches dérivent des souches $\mathrm{Nal}^{\mathrm{R}}-\mathrm{Cip}^{\mathrm{SD}}$ par acquisition de mutations chromosomiques additionnelles (une deuxième mutation dans le gène gyrA et une mutation dans le gène parC codant pour une sous-unité de la topoisomérase IV, cible secondaire des quinolones) [32] ou bien par acquisition d'un gène plasmidique de résistance aux quinolones appelé qnr [33]. En dehors du problème thérapeutique qu'elles posent (aucune fluoroquinolone ne peut être active), ces souches ne présentent pas de difficulté d'identification au niveau du laboratoire contrairement aux souches $C_{i p}{ }^{S D}$. Des souches résistantes aux C3G ont déjà été décrites depuis 2008 [34-36]. II s'agit de souches asiatiques (Philippines, Irak et Inde) produisant des bêtalactamases à spectre étendu de type CTX-M-15 ou SHV-12. Cependant, elles restent très rares : seulement 2 souches de ce type ont été détectées sur les 2000 souches de Salmonella Typhi isolées aux États-Unis entre 1999 et 2006 [25]. Concernant la résistance à l'azithromycine, il n'existe pas encore à l'heure actuelle de concentrations critiques définies par les sociétés savantes pour cet antibiotique. Cependant, aucune souche potentiellement résistante n'a encore été documentée au CNR (CMI $\leq 8 \mathrm{mg} /$ I pour 104 souches Cip ${ }^{S}$ ou Cip ${ }^{\text {SD }}$ isolées en 2009) ou dans la littérature, à l'exception d'une souche $\operatorname{Cip}^{R}$ indienne pour laquelle la CMI de l'azithromycine était de $64 \mathrm{mg} / \mathrm{I}$ [29, 37, 38]. L'utilisation croissante de cet antibiotique dans cette indication étant inéluctable, il est prévisible que de telles souches résistantes émergent assez rapidement. Un parallèle peut être fait avec l'apparition de souches de Shigella sonnei résistantes à l'azithromycine deux ans et demi après la préconisation de cet antibiotique dans le traitement des shigelloses à S. sonnei de l'enfant en France [39].

La poursuite de la surveillance microbiologique de cet agent infectieux, et notamment la publication de données nationales régulières concernant sa sensibilité aux antibiotiques en fonction du pays de contamination est donc un élément-clé pour l'établissement de bonnes recommandations thérapeutiques. Les difficultés croissantes dans la lutte chimiothérapique contre ces bactéries doivent conduire à un renforcement des mesures préventives (règles d'hygiène, vaccination) tant sur le plan individuel qu'au niveau de la collectivité. $\diamond$

\section{SUMMARY}

Typhoid fever:

facing the challenge of resistant strains

The introduction of chloramphenicol in 1948 revolutionised the outcome of typhoid fever but chloramphenicol-resistant strains of Salmonella enterica serotype Typhi were reported just two years later. Resistance followed also the introduction of ampicillin and cotrimoxazole. During the second half of the 1980s, strains resistant to the three first-line antimicrobial agents, chloramphenicol, ampicillin and co-trimoxazole emerged and spread rapidly throughout the Indian subcontinent and South East Asia. During the 1990s when fluoroquinolones had become a first-line treatment for typhoid fever, these multi drug resistant (MDR) strains acquired an additional resistance to nalidixic acid with decreased susceptibilities to ciprofloxacin (CIPDS) (MIC range, 0.125-1 mg/l). Considerable data have now accumulated to suggest that infections due to CIPDS strains may not respond satisfactorily to therapy with ciprofloxacin or ofloxacin. Furthermore, identification of such CIPDS strains in clinical laboratories is not easy without determination of MIC of ciprofloxacin. Recently, several isolates highly resistant to ciprofloxacin or to extended-spectrum cephalosporins of Asian origin have been reported. $\diamond$

\section{CONFLIT D'INTÉRÊTS}

L'auteur déclare n'avoir aucun conflit d'intérêts concernant les données publiées dans cet article.

\section{RÉFÉRENCES}

1. Roumagnac P, Weill FX, Dolecek C, et al. Evolutionary history of Salmonella Typhi. Science 2006 ; 314 : 1301-4.

2. Delmas G, Haeghebaert S, de Valk J, Weill FX. Les fièvres typhoïdes et paratyphoïdes en France de 2001 à 2003. Surveillance nationale des maladies infectieuses. URL : http://www.invs.sante.fr/publications/2005/ snmi/pdf/fievres_typhoides.pdf.

3. Crump JA, Luby SP, Mintz ED. The global burden of typhoid fever. Bull World Health Organ $2004 ; 82: 346-53$.

4. Anderson ES. The problem and implications of chloramphenicol resistance in the typhoid bacillus. J Hyg (Lond) $1975 ; 74: 289-99$.

5. Anderson ES, Smith HR. Chloramphenicol resistance in the typhoid bacillus. Br Med J $1972 ; 3: 329-31$.

6. Olarte J, Galindo E. Salmonella typhi resistant to chloramphenicol, ampicillin, and other antimicrobial agents: strains isolated during an extensive typhoid fever epidemic in Mexico. Antimicrob Agents Chemother $1973 ; 4:$ 597-601.

7. Datta N, Olarte J. R factors in strains of Salmonella typhi and Shigella dysenteriae 1 isolated during epidemics in Mexico: classification by compatibility. Antimicrob Agents Chemother $1974 ; 5$ : 310-7.

8. Meyruey MH, Goudineau JA, Berger P, et al. Actualité de la fièvre typhoïde au Sud Vietnam. Rev Epidemiol Med Soc Santé publique 1975 ; 23 : 345-58. 
9. Chabbert YA, Gerbaud GR. Surveillance épidémiologique des plasmides responsables de la résistance au chloramphénicol de Salmonella typhi. Ann Microbiol (Paris) 1974 ; 125A : 153-66.

10. Goldstein FW, Chumpitaz JC, Guevara JM, et al. Plasmid-mediated resistance to multiple antibiotics in Salmonella typhi. J Infect Dis 1986 ; $153: 261-6$.

11. Wain J, Kidgell C. The emergence of multidrug resistance to antimicrobial agents for the treatment of typhoid fever. Trans R Soc Trop Med Hyg $2004 ; 98: 423-30$

12. Le TA, Lejay-Collin M, Grimont PA, et al. Endemic, epidemic clone of Salmonella enterica serovar typhi harboring a single multidrug-resistant plasmid in Vietnam between 1995 and 2002. J Clin Microbiol $2004 ; 42$ : 3094-9.

13. Threlfall $\varepsilon$ J, Ward LR. Decreased susceptibility to ciprofloxacin in Salmonella enterica serotype Typhi, United Kingdom. Emerg Infect Dis $2001 ; 7: 448-50$.

14. Le TA, Fabre L, Roumagnac $P$, et al. Clonal expansion and microevolution of quinolone resistant Salmonella enterica serotype Typhi in Vietnam from 1996 to 2004. J Clin Microbiol 2007 ; $45: 3485-92$.

15. Wain J, Hoa NT, Chinh NT, et al. Quinolone-resistant Salmonella typhi in Viet Nam: molecular basis of resistance and clinical response to treatment. Clin Infect Dis 1997 ; 25 : 1404-10.

16. Crump JA, Barrett TJ, Nelson JT, Angulo FJ. Reevaluating fluoroquinolone breakpoints for Salmonella enterica serotype Typhi and for non-Typhi salmonellae. Clin Infect Dis 2003 ; $37: 75-81$.

17. Aarestrup FM, Wiuff C, Mølbak K, Threlfall $\varepsilon$ J. Is it time to change fluoroquinolone breakpoints for Salmonella spp.? Antimicrob Agents Chemother 2003 ; 47 : 827-9.

18. Crump JA, Kretsinger K, Gay K, et al. Clinical response and outcome of infection with Salmonella enterica serotype Typhi with decreased susceptibility to fluoroquinolones: a United States foodnet multicenter retrospective cohort study. Antimicrob Agents Chemother 2008 ; 52 : 1278-84.

19. Booker BM, Smith PF, Forrest A, et al. Application of an in vitro infection model and simulation fo reevaluation of fluoroquinolone breakpoints for Salmonella enterica serotype typhi. Antimicrob Agents Chemother $2005 ; 49: 1775-81$.

20. Smith MD, Duong NM, Hoa NT, et al. Comparison of ofloxacin and ceftriaxone for short-course treatment of enteric fever. Antimicrob Agents Chemother 1994 ; 38 : 1716-20.

21. Tran TH, Bethell DB, Nguyen $\Pi$, et al. Short course of ofloxacin for treatment of multidrugresistant typhoid. Clin Infect Dis $1995 ; 20: 917-23$.

22. Nguyen TC, Solomon T, Mai XT, et al. Short courses of ofloxacin for the treatment of enteric fever. Trans R Soc Trop Med Hyg 1997 ; 91 : 347-9.

23. Weill FX, Tran HH, Roumagnac $P$, et al. Clonal reconquest of antibiotic-susceptible Salmonella enterica serotype Typhi in Son La Province, Vietnam. Am J Trop Med Hyg 2007 ; 76 : 1174-81.

24. Cooke FJ, Day M, Wain J, et al. Cases of typhoid fever imported into England, Scotland and Wales (2000-2003). Trans R Soc Trop Med Hyg 2007 ; 101 : 398-404.

25. Lynch MF, Blanton EM, Bulens S, et al. Typhoid fever in the United States, 1999-2006. JAMA 2009; $302: 859-65$.

26. Chau T, Campbell JI, Galindo CM, et al. Antimicrobial drug resistance of Salmonella enterica serovar Typhi in asia and molecular mechanism of reduced susceptibility to the fluoroquinolones. Antimicrob Agents Chemother 2007 ; $51: 4315-23$

27. Gaborieau V, Weill FX, Marchou B. Salmonella enterica serovar Typhi de sensibilité diminuée à la ciprofloxacine. Med Mal Infect 2010 (sous presse).
28. Song $Y$, Roumagnac P, Weill FX, et al. A multiplex single nucleotide polymorphism typing assay for detecting mutations that result in decreased fluoroquinolone susceptibility in Salmonella enterica serovars Typhi and Paratyphi A. J Antimicrob Chemother 2010; 65 : 1631-41.

29. Parry CM, Ho VA, Phuong le T, et al. Randomized controlled comparison of ofloxacin, azithromycin, and an ofloxacin-azithromycin combination for treatment of multidrug-resistant and nalidixic acid-resistant typhoid fever. Antimicrob Agents Chemother 2007 ; $51: 819-25$.

30. Pandit A, Arjyal A, Day JN, et al. An open randomized comparison of gatifloxacin versus cefixime for the treatment of uncomplicated enteric fever. PLoS One 2007 ; 2 : e542.

31. Dolecek C, Tran TP, Nguyen NR, et al. A multi-center randomised controlled trial of gatifloxacin versus azithromycin for the treatment of uncomplicated typhoid fever in children and adults in Vietnam. PLoS One 2008 ; 3 : e2188.

32. Dimitrov T, Dashti AA, Albaksami 0, et al. Ciprofloxacin-resistant Salmonella enterica serovar Typhi from Kuwait with novel mutations in gyrA and parC genes. J Clin Microbiol 2009; 47 : 208-11.

33. Keddy KH, Smith AM, Sooka A, et al. Fluoroquinolone-resistant typhoid, South Africa. Emerg Infect Dis $2010 ; 16: 879-80$.

34. Rotimi VO, Jamal W, Pal T, et al. Emergence of CTX-M-15 type extendedspectrum beta-lactamase-producing Salmonella spp. in Kuwait and the United Arab Emirates. J Med Microbiol 2008 ; 57 : 881-6.

35. Al Naiemi N, Zwart B, Rijnsburger MC, et al. Extended-spectrum-betalactamase production in a Salmonella enterica serotype Typhi strain from the Philippines. J Clin Microbiol 2008; 46 : 2794-5.

36. Pfeifer Y, Matten J, Rabsch W. Salmonella enterica serovar Typhi with CTX-M beta-lactamase, Germany. Emerg Infect Dis 2009 ; 15 : 1533-5.

37. Capoor MR, Rawat D, Nair D, et al. In vitro activity of azithromycin, newer quinolones and cephalosporins in ciprofloxacin-resistant Salmonella causing enteric fever. J Med Microbiol 2007 ; 56 : 1490-4.

38. Threlfall $\varepsilon$ J, de Pinna $\varepsilon$, Day $M$, et al. Alternatives to ciprofloxacin use for enteric fever, United Kingdom. Emerg Infect Dis 2008; 14 : 860-1.

39. Boumghar-Bourtchai L, Mariani-Kurkdjian P, Bingen $\varepsilon$, et al. Macrolideresistant Shigella sonnei. Emerg Infect Dis 2008 ; 14 : 1297-9.

40. Bhutta ZA. Current concepts in the diagnosis and treatment of typhoid fever. BMJ $2006 ; 333: 78-82$.
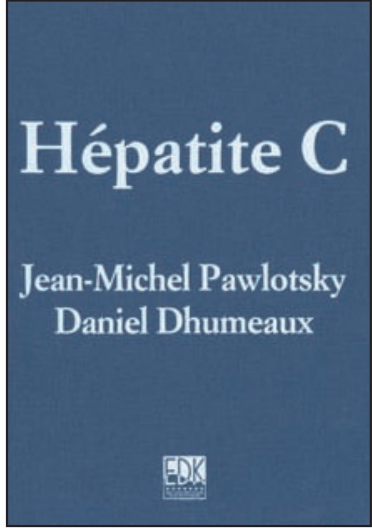

ISBN : 2-84254-096-4 512 pages

\section{Bon de commande}

À retourner à EDK, 2, rue Troyon - 92316 Sèvres Cedex

Tél. : 0155641393 - Fax : 0155641394 - E-mail : edk@edk.fr

NOM : Prénom :

Adresse

Code postal :

Ville :

Pays :

Fonction :

Je souhaite recevoir l'ouvrage Hépatite C : $65 €+3 €$ de port $=\mathbf{6 8} €$ TTC

en ................. exemplaire, soit un total de ......................................... €

$\square$ Par chèque, à l'ordre de $\mathbf{E} \mathbf{D} \mathbf{K}$

$\square$ Par carte bancaire : $\square$ Visa $\square$ Eurocard/Mastercard

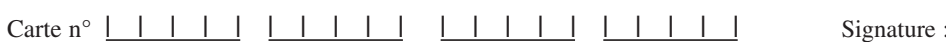

Date d'expiration: $\quad|\quad| \quad|\quad| \quad \mid$

$N^{\circ}$ de contrôle au dos de la carte : $\quad|\quad| \quad \mid$
TIRÉS À PART

F.X. Weill 